\title{
Tunable blue light source by intracavity frequency doubling of a $\mathrm{Cr}$-doped LiSrAlF $_{6}$ laser
}

François Balembois, Patrick Georges, François Salin, Gerard Roger, and Alain Brun

Institut d'Optique Théorique et Appliquée, Unité de Recherche Associee au CNRS $N^{\circ} 14$, Université Paris-Sud, B.P. 147, 91403 Orsay Cedex, France

(Reccived 13 July 1992; accepted for publication 11 September 1992)

$\mathrm{A} \mathrm{Cr}^{3+}: \mathrm{LiSrAlF}_{6}$ laser in Q-switched operation at $10 \mathrm{kHz}$ was intracavity frequency doubled by using a $\mathrm{LiIO}_{3}$ crystal. The 230 ns tunable blue pulses were obtained in the $395-435 \mathrm{~nm}$ range with up to $7 \mathrm{~mW}$ average power at $407 \mathrm{~nm}$.

Compact solid state lasers emitting in the blue-green wavelength range are expected to be the key components for optical recording and underwater communications or as spectroscopic sources. To develop such lasers, several approaches are under study at numerous laboratories around the world. Possible solutions include up-conversion lasers, ${ }^{1,2}$ green or blue diode lasers, ${ }^{3}$ sum frequency mixing techniques $^{4}$ or frequency doubling near-IR sources ${ }^{5}$ that produce blue or green light at fixed wavelengths. Compact sources generating tunable blue light could be particularly useful for spectroscopic experiments. One possibility is an optical parametric oscillator pumped by the third harmonic of a YAG laser but this solution requires high peak power from the pump laser. ${ }^{6}$

In this letter, we report on a tunable blue source using an intracavity frequency doubled, Q-switched, cw pumped, $\mathrm{Cr}^{3+}: \mathrm{LiSrAlF}_{6}$ (Cr:LiSAF) laser. $\mathrm{Cr}^{3+} \mathrm{LiSrAlF}_{6}$ was discovered in 1989 by Payne et al. at the Lawrence Livermore National Laboratory. ${ }^{7}$ It has an absorption band in the red between 600 and $700 \mathrm{~nm}$ and can be pumped by red diode lasers. ${ }^{8}$ Its large fluorescence bandwidth leads to tunable laser emission between 750 and $1000 \mathrm{~nm} .^{9}$ A compact tunable blue laser source can thus be obtained by intracavity second harmonic generation of a Cr:LiSAF laser.

In order to simulate diode pumping, we used the red lines (647 and $676 \mathrm{~nm}$ ) of a cw krypton ion laser to pump our $15 \mathrm{~mm}$ long Cr:LiSAF crystal. The $\mathrm{Cr}^{3+}$ concentration was $0.8 \%$ in weight and the absorption was $97 \%$ of the pump power. Cr:LiSAF has a relatively long fluorescence life time $(67 \mu \mathrm{s})$ as compared to titanium sapphire $(3 \mu \mathrm{s})$ and a large saturation fluence $\left(4.7 \mathrm{~J} / \mathrm{cm}^{2}\right.$ at 840 $\mathrm{nm}) .{ }^{1}$ It is therefore suitable for the generation of high energy nanosecond pulses in Q-switched operation. The pulses were obtained by modulating the intracavity losses with an acousto-optic crystal operating at $125 \mathrm{MHz}$. The cavity mode spacing was $190 \mathrm{MHz}$, which means that the acousto-optic modulator did not work as a modelocker (requiring $250 \mathrm{MHz}$ cavity mode spacing), but rather as a loss source. At a repetition rate of a few $\mathrm{kHz}$, we switched off the rf power and thereby decreased the losses and allowed the laser to produce nanosecond pulses.

The laser configuration is shown in Fig. 1. The krypton ion laser beam was focused by a $10 \mathrm{~cm}$ focal lens through a dichroic mirror $M_{1}$ into the Cr:LiSAF crystal. This 10 $\mathrm{cm}$ radius of curvature mirror had a high reflectivity between 800 and $900 \mathrm{~nm}$ and a high transmission in the 600-700 $\mathrm{nm}$ range. The length between $M_{1}$ and the lens was adjusted so that the pump beam matched the infrared beam in size and divergence as closely as possible. The infrared spot had a diameter of $60 \mu \mathrm{m}$ in the $\mathrm{Cr}$ :LiSAF crystal. $\boldsymbol{M}_{2}$ was a $10 \mathrm{~cm}$ radius of curvature mirror with high reflectivity between 800 and $900 \mathrm{~nm} . M_{3}$ was a plane output coupler with $22.5 \%$ transmission in the same wavelength range. The cavity consisted of the three mirrors, $M_{1}$, $M_{2}$, and $M_{3}$, and was calculated to compensate the astigmatism introduced by our brewster end cut $\mathrm{Cr}$ :LiSAF crystal. A birefringent filter was used to tune the laser wavelength. Cr:LiSAF, in comparison to titanium sapphire, is of relatively poor thermomechanical quality and we observed thermal damage to our sample for $2 \mathrm{~W} \mathrm{cw}$ pump power. Therefore, for higher pumping rates, the krypton laser was chopped at $200 \mathrm{~Hz}$ with a $25 \%$ duty cycle.

With an output coupler of $22.5 \%$ transmission at 830 $\mathrm{nm}$, this LiSAF laser produced pulses with a duration of $100 \mathrm{~ns}$ and an energy of $26 \mu \mathrm{J}$ at a repetition rate around $10 \mathrm{kHz}$ for $3.3 \mathrm{~W}$ pump power incident on the crystal. The energy per pulse was constant for frequencies up to $14 \mathrm{kHz}$ and decreased above this value. This roll-off point is consistent with the $67 \mu$ s lifetime of Cr:LiSAF. We can tune the laser over $100 \mathrm{~nm}$, between 780 and $880 \mathrm{~nm}$ (Fig. 2). The infrared limit is given by the dielectric coatings of the mirrors. The pulse length remained within 100 and $180 \mathrm{~ns}$ with an energy higher than $10 \mu \mathrm{J}$ over the entire range.

The high peak power obtained $(260 \mathrm{~W}$ at $830 \mathrm{~nm})$ allows efficient intracavity second harmonic generation. We therefore modified the laser configuration (see Fig. 3) and introduced a $1 \mathrm{~mm}$ long $\mathrm{LiIO}_{3}$ crystal at the focal

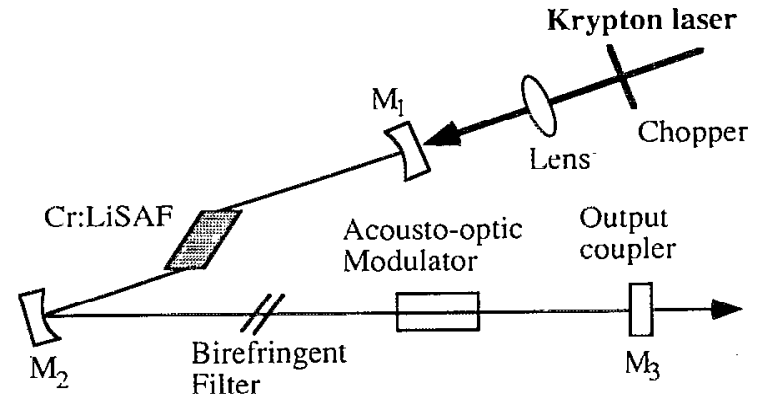

FIG. 1. Experimental set-up $M_{1}$ and $M_{2}$ are $R=100 \mathrm{~mm}$ HR mirrors. $M_{3}$ is a plane mirror with a transmission of $22.5 \%$. 


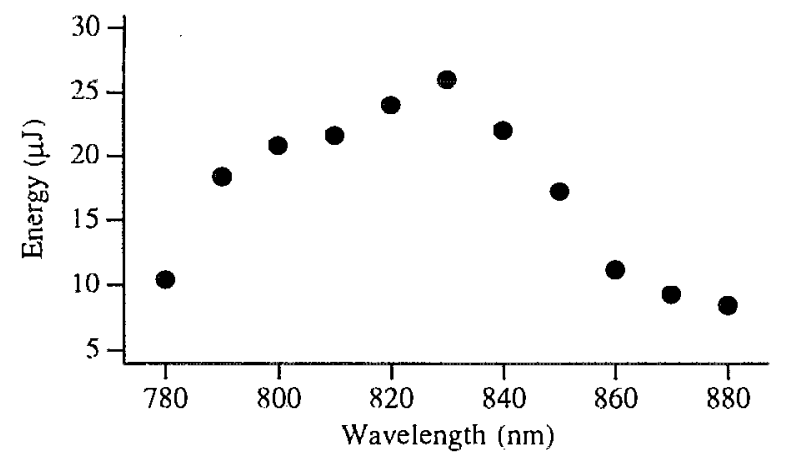

FIG. 2. Tunability in Q-switch operation at $3.3 \mathrm{~W}$ pump power. Repetition rate is $10 \mathrm{kHz}$.

point of a second cavity formed by two $15 \mathrm{~cm}$ radius of curvature mirrors $M_{4}$ and $M_{5}$. The infrared spot had a size of $100 \mu \mathrm{m}$ in the nonlinear crystal. The mirrors were dichroic coated to allow maximum transmission in the blue. They had a high reflectivity between 760 and $830 \mathrm{~nm}$. Blue light could be observed on each mirror of this second cavity. Counting both outputs, we obtained blue pulses of 0.74 $\mu \mathrm{J}$ at $407 \mathrm{~nm}$ at a repetition rate of $10 \mathrm{kHz}$. This corresponds to $7.4 \mathrm{~mW}$ average blue power at $3.3 \mathrm{~W}$ pump power incident on the Cr:LiSAF crystal. The infrared pulses were $280 \mathrm{~ns}$ long (the increase in duration is due to more important intracavity losses) while the blue pulses were $230 \mathrm{~ns}$ long at $407 \mathrm{~nm}$. The decrease in pulse length is consistent with the fact that the second harmonic intensity is proportional to the square of the fundamental intensity. We could tune the blue emission wavelength by simultaneously adjusting the birefringent filter to vary the infrared wavelength and modifying the $\mathrm{LiIO}_{3}$ crystal orientation to preserve phase matching. We observed blue light generation between 395 and $435 \mathrm{~nm}$ (Fig. 4). The decrease around $415 \mathrm{~nm}$ is due to the cavity mirrors as their transmission increases above $830 \mathrm{~nm}$. This leads to a decrease in energy in the cavity and a corresponding decrease in blue generation.

Several possibilities for improving this laser exist. With better adapted mirrors, it should be possible to tune the

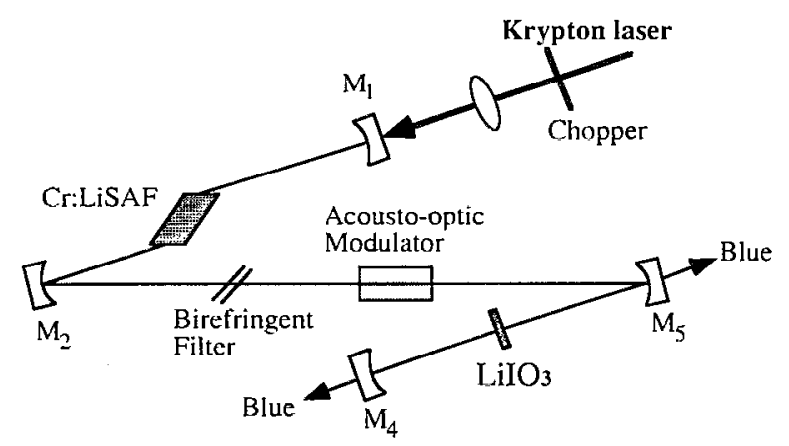

FIG. 3. Experimental set-up for intracavity second harmonic generation. $M_{4}$ and $M_{5}$ are $R=150 \mathrm{~mm}$.

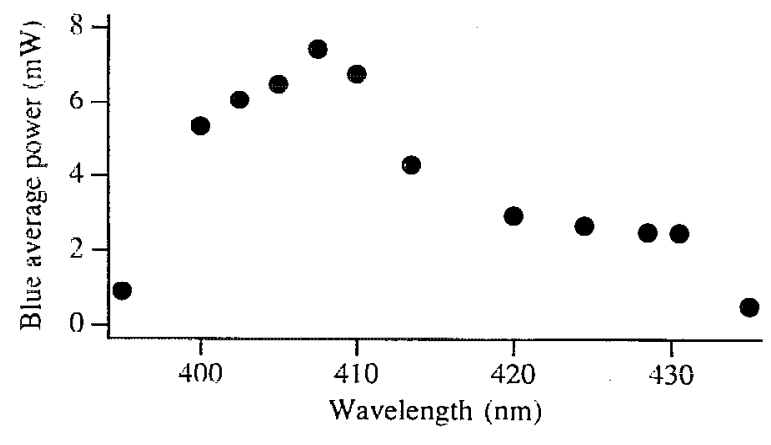

FIG. 4. Tunability of blue emission at $3.3 \mathrm{~W}$ pump power. Repetition rate is $10 \mathrm{kHz}$.

laser between 390 and $500 \mathrm{~nm}$, corresponding to the entire emission band of the Cr:LiSAF crystal. We also considered the effect on the efficiency of blue generation of the infrared spot size on the $\mathrm{LiIO}_{3}$ crystal. We tried two other configurations with different radii of curvature for the mirrors of the second cavity leading to spot sizes of 70 and $200 \mu \mathrm{m}$. However these configurations lead to a weaker output of blue than the initial one. Our experiment could be improved by minimizing intracavity losses through the use of a nonlinear crystal with antireflection coatings in both the blue and the infrared range. Finally, it could be modified so as to have only one blue output by introducing a flat mirror between the two cavities (HR around $830 \mathrm{~nm}$ and HT around $415 \mathrm{~nm}$ ) and using two mirrors with HR at 830 and $415 \mathrm{~nm}$ mirrors for the second cavity.

In conclusion, we describe a blue laser source with 40 $\mathrm{nm}$ tunability $(395-435 \mathrm{~nm}$ ) producing up to $7 \mathrm{~mW}$ average power around $407 \mathrm{~nm}$. We believe we could reach 10 $\mathrm{mW}$ average power by using mirrors with optimized coatings and by using more efficient nonlinear crystals such as $\mathrm{KNbO}_{3}$ in place of our $\mathrm{LiIO}_{3}$ crystal. Many applications such as optical data storage or spectroscopy of biological media require around $10 \mathrm{~mW}$ of blue laser light. Thus, once the krypton pump laser is replaced by laser diodes, this source will be suitable for a number of sensors and instruments.

We thank B. Deveaud (CNET Lannion) for the loan of the $\mathrm{LiIO}_{3}$ crystal.

' J. Y. Allain, M. Monerie, and H. Poignant, Electron Lett. 26, 166 (1990).

${ }^{2}$ R. M. Mcfarlane, F. Tong, A. J. Silversmith, and W. Lenth, Appl. Phys. Lett. 52, 1300 (1988).

${ }^{3}$ M. A. Hanse, J. Qiu, J. M. Depuydt, and H. Cheng, Appl. Phys. Lett. 59, 1272 (1991).

${ }^{4}$ J. C. Baumert, F. M. Schettenberg, W. Lenth, W. P. Risk, and G. C. Bjorklund, Appl. Phys. Lett. 51, 2192 (1987).

${ }^{5}$ G. J. Dixon, C. E. Tanner, and C. E. Wieman, Opt. Lett. 14, 731 (1989).

'J. T. Lin, Opt. Quantum Electron. 22, S283 (1990).

${ }^{7}$ S. A. Payne, L. L. Chase, L. K. Smith, W. L. Kway, and H. W. Newkirk, J. Appl. Phys. 66, 1051 (1989).

${ }^{8}$ R. Shep, J. F. Myers, H. B. Seneze, A. Resenberg, R. C. Morris, and M. Long, Opt. Lett. 16, 820 (1991).

${ }^{9}$ M. Stalder, B. H. T. Chai, and M. Bass, Appl. Phys. Lett. 58, 216 (1991). 